\title{
Eradication of Neuroblastoma by T Cells Redirected with an Optimized GD2-Specific Chimeric Antigen Receptor and Interleukin-15
}

\author{
Yuhui Chen', Chuang Sun', Elisa Landoni', Leonid Metelitsa ${ }^{2}$, Gianpietro Dotti ${ }^{1,3}$, and \\ Barbara Savoldo ${ }^{1,4}$
}

\begin{abstract}
Purpose: A delay in encountering the cognate antigen while in the circulation, and the suboptimal costimulation received at the tumor site are key reasons for the limited activity of chimeric antigen receptor-redirected T cells (CAR-T) in solid tumors. We have explored the benefits of incorporating the IL15 cytokine within the CAR cassette to provide both a survival signal before antigen encounter, and an additional cytokine signaling at the tumor site using a neuroblastoma tumor model.

Experimental Design: We optimized the construct for the CAR specific for the NB-antigen GD2 without (GD2.CAR) or with IL15 (GD2.CAR.15). We then compared the expansion, phenotype, and antitumor activity of $\mathrm{T}$ cells transduced with these constructs against an array of neuroblastoma cell lines in vitro and in vivo using a xenogeneic metastatic model of neuroblastoma.
\end{abstract}

\section{Introduction}

Neuroblastoma is the most common extracranial tumor of childhood (1). Because children with high-risk neuroblastoma continue to have poor outcome despite intensive chemotherapy regimens, new treatment strategies are required $(2,3)$. The disialoganglioside GD2, which is consistently expressed in neuroblastoma tumor cells with restricted expression in normal tissues, represents the target of choice for immunotherapy in neuroblastoma, through either a specific mAb (Ch14.18, dinutuximab) or, more recently, chimeric antigen receptor engineered T cells (GD2. CAR-Ts) (3-6).

In the first clinical study with GD2.CAR-Ts, the specific scFv was derived from the murine 14.G2a mAb coupled with the $\zeta$-chain endodomain (first-generation CAR), and engrafted in EpsteinBarr virus-specific CTLs $(5,6)$. In the second clinical trial, the same

'Lineberger Comprehensive Cancer Center, University of North Carolina at Chapel Hill, Chapel Hill, North Carolina. ${ }^{2}$ Department of Pediatrics, Texas Children's Hospital, Houston, Texas. ${ }^{3}$ Department of Microbiology and Immunology, University of North Carolina at Chapel Hill, Chapel Hill, North Carolina. ${ }^{4}$ Department of Pediatrics, University of North Carolina at Chapel Hill, Chapel Hill, North Carolina.

Note: Supplementary data for this article are available at Clinical Cancer Research Online (http://clincancerres.aacrjournals.org/).

Corresponding Author: Barbara Savoldo, University of North Carolina at Chapel Hill, 125 Mason Farm Road, Marsico Hall 5203, Chapel Hill, NC 27599. Phone: 919 962-8414; Fax: 919-843-9107; E-mail: bsavoldo@med.unc.edu

doi: 10.1158/1078-0432.CCR-18-1811
Results: We observed that optimized GD2.CAR.15-Ts have reduced expression of the PD-1 receptor, are enriched in stem cell-like cells, and have superior antitumor activity upon repetitive tumor exposures in vitro and in vivo as compared with GD2.CAR-Ts. Tumor rechallenge experiments in vivo further highlighted the role of IL15 in promoting enhanced CAR-T antitumor activity and survival, both in the peripheral blood and tissues. Finally, the inclusion of the inducible caspase-9 gene (iC9) safety switch warranted effective on demand elimination of the engineered GD2. CAR.15-Ts.

Conclusions: Our results guide new therapeutic options for GD2.CAR-Ts in patients with neuroblastoma, and CAR-T development for a broad range of solid tumors.

CAR was modified to encode two costimulatory endodomains in tandem (CD28 and OX40) (third-generation CAR) and expressed in polyclonal T lymphocytes (4). Both studies showed that infusions of GD2.CAR-Ts were well tolerated, even when combined with conditioning regimens to lymphodeplete the host, and promoted some objective clinical responses (4-6).

However, response rates in patients with neuroblastoma receiving GD2.CAR-Ts remain significantly inferior to those observed in patients with acute lymphoblastic leukemia treated with CD19specific CAR-Ts (7). It is also evident that, although the inclusion of costimulatory endodomains like CD28 or 4-1BB in the CD19. specific CAR is necessary and sufficient to achieve clinical responses (7-11), CAR costimulation is not equally effective in neuroblastoma (4). Furthermore, although the expansion of CD19-specific CAR-Ts in the peripheral blood correlates with clinical responses (7), increases of GD2.CAR-Ts in the peripheral blood, even when these cells are infused in lymphodepleted patients, do not promote better antitumor responses (4). We speculated that, despite homeostatic expansion in peripheral blood, in contrast to CD19-specific CAR-Ts, GD2.CAR-Ts do not promptly receive the costimulation required for full activation while in the circulation, as they do not engage their cognate antigen. Furthermore, when GD2.CAR-Ts reach the tumor site, the costimulation provided through the signaling moieties of the CAR remains suboptimal to achieve complete elimination of tumor cells due to local inhibitory/suppressive factors. We observed here that the inclusion of the IL15 cytokine within the CAR cassette is beneficial for GD2.CAR-Ts by promoting both enhanced survival in the absence of the cognate antigen, and prolonged antitumor activity. 


\section{Translational Relevance}

Response rates in patients with neuroblastoma receiving GD2.CAR-Ts remain significantly inferior to those observed in patients with acute lymphoblastic leukemia treated with CD19.CAR-Ts. This is consequent to the limited GD2.CAR-Ts survival in the circulation and at the tumor site. To solve these issues, we have incorporated IL15 within the CAR construct to generate GD2.CAR.15-Ts. These cells enriched in central memory/stem cell-like cells and expressing PD-1 at lower level promoted superior antitumor activity, expansion, and survival in vitro and in vivo. To improve the safety of the GD2. CAR.15-Ts, we have incorporated the iC9 safety switch gene and validated the feasibility of generating T-cell products incorporating the three genes in accordance with good manufacturing practice. Our discoveries guide an efficient and safe therapeutic option of GD2.CAR-Ts in patients with neuroblastoma, and are highly relevant for the development of CAR-T for solid tumors in general.

\section{Materials and Methods}

\section{Cell lines}

The neuroblastoma cell lines IMR-32, LAN-1, and SKNLP were purchased from ATCC, while the CHLA255 cell line was described previously (12). Cells were maintained in RPMI1640 supplemented with $10 \%$ FBS and $2 \mathrm{mmol} / \mathrm{L}$ L-glutamine (Invitrogen). Tumor cell lines were transduced with a gamma retroviral vector encoding the enhanced GFP (eGFP) or the Firefly-luciferase (FFLuc) genes as described previously (13). Cells were routinely (monthly) screened for the presence of Mycoplasma and expression of the GD2 antigen and kept in culture for less than 3 consecutive months, after which aliquots from the original expanded vial were used.

\section{Plasmid and retrovirus construction}

The cassette encoding the GD2-specific scFv (scFv14G2a), the CD $8 \alpha$ stalk and transmembrane domain, the CD28 intracellular domain and $\mathrm{CD} 3 \zeta$ chain was cloned into the SFG backbone (GD2.CAR). The scFv14G2a was previously identified (14) and used in clinical studies (4-6). We modified this scFv14G2a by removing 16 amino acids from the $\mathrm{COOH}$-region of the $\mathrm{V}_{\mathrm{H}}$ (AKTTPPSVYGRVYVSS). Indeed, when we aligned the previously used $\mathrm{V}_{\mathrm{H}}$ sequence into the IGBLAST NCBI database, these 16 amino acids, which belong to the CDR3-IMGT (germline) of the $\mathrm{V}_{\mathrm{H}}$, exceeded the alignment of the $\mathrm{V}_{\mathrm{H}}$ sequence reported in the dataset. Furthermore, two versions of the scFv14G2a were produced by conserving the previously used 9-aminoacid linker (SL) between the $V_{L}$ and $V_{H}$ fragments or including a 20-aminoacid linker (LL). We then generated vector cassettes encoding the CAR in combination with the IL-15 $c d n a$ (GD2.CAR.15) using a 2Asequence peptide (15). In a third vector, we also cloned the inducible caspase-9 suicide gene (16) into the GD2.CAR.15 using a second 2A-sequence peptide (iC9.GD2.CAR.15). Finally, for selected experiments, the scFv14G2a in the iC9.GD2.CAR.15 vector was swapped with the CD19-specific scFv (17) to obtain the iC9.CD19.CAR.IL-15 vector. Vectors encoding the Firefly-luciferase (FFluc) and the fusion protein GFP have been previously described (13). The full-length human PD-L1
(accession_NM_014143.4) was PCR amplified, cloned into the retroviral vector SFG and used to transduce the CHLA255 tumor cell line to generate tumor cells constitutively expressing high levels of PD-L1 (CHLA255-PD-L1 ${ }^{+}$). Transient retroviral supernatants were produced as previously described (13). For GMP translation and validation studies a clinical grade packaging cell line was generated by using the PG13 packaging cell line (gibbon ape leukemia virus pseudotyping packaging cell line; CRL-10686, ATCC) as previously described (5). We used the highest titer clone to establish the master cell bank as recommended by FDA guidelines.

\section{Generation of CAR-Ts}

Peripheral blood mononuclear cells (PBMC) were isolated from buffy coats (Gulf Coast Regional Blood Center) using Lymphoprep (Accurate Chemical and Scientific Corporation). PBMCs were then activated using $1 \mu \mathrm{g} / \mathrm{mL}$ immobilized CD3 (Miltenyi Biotec) and CD28 (BD Biosciences) mAbs. After 3 days, cells were transduced with retroviral supernatants and expanded in complete medium (45\% RPMI1640 and 45\% Click's medium, $10 \% \mathrm{FBS}$, and $2 \mathrm{mmol} / \mathrm{L}$ GlutaMAX) supplemented with IL7 (10 ng/mL, PeproTech) and IL15 (5 ng/mL, PeproTech) and expanded by feeding them every 3 to 4 days with fresh complete media and cytokines, as described previously (18-20).

\section{Western blot analysis}

T-cell lysates were resuspended in $2 \times$ Laemelli Buffer (Bio-Rad) in reducing (with $\beta$-mercaptoethanol) or nonreducing (without $\beta$-mercaptoethanol) conditions. All lysates were separated in $4 \%$ to $15 \%$ SDS-PAGE gels and transferred to polyvinylidene diflouride membranes (all Bio-Rad). Blots were probed for human CD3 $\zeta$ $\mathrm{Ab}$ (Santa Cruz Biotechnology) diluted 1:1,000 in TBS-Tween/5\% skim milk. Membranes were then incubated with HRP-conjugated goat $\alpha$-mouse or goat $\alpha$-rabbit IgG (both Santa Cruz Biotechnology) at a dilution of 1:3,000 and imaged using the ECL Substrate Kit on a ChemiDoc MP System (both BioRad) according to the manufacturer's instructions.

\section{Coculture experiments}

eGFP-transduced tumor cells were plated in 24-well plates at a concentration of 0.25 or $0.5 \times 10^{6}$ cells/well the day before the addition of T cells, in the absence of cytokines. Different effector cells to target cells (E:T) ratios were used (1:1, 1:5 and 1:10). For the repetitive coculture experiments, $\mathrm{T}$ cells and eGFP-tagged neuroblastoma cells were cocultured at the E:T ratio of $1: 1$ or 1:5. At day 3, all cells were transferred into a new well and seeded with $0.25 \times 10^{6}$ neuroblastoma cells. T cells and neuroblastoma cells were quantified by flow cytometry after 4 cycles based on CD3 and GFP expression, respectively. Supernatant were also collected for cytokine measurements after each cycle. For each experiment, nontransduced $\mathrm{T}$ cells were used as a negative control (Ctrl-Ts).

\section{Carboxyfluorescein succinimidyl ester-based proliferation assay}

CAR-Ts and control T cells were labeled with carboxyfluorescein succinimidyl ester (CFSE; Invitrogen) and plated in the wells precoated with or without the $1 \mathrm{~A} 7$ antibody $(0.5 \mu \mathrm{g} /$ well $)$. On day 5 , cells were collected and analyzed for CFSE dilution by flow cytometry. 
Side population staining by Hoechst dye

Cells were incubated in Hank's Balanced Salt Solution (Life Technologies) containing 2\% FBS, $10 \mathrm{mmol} / \mathrm{L}$ HEPES (Life Technologies), and either $10 \mu \mathrm{g} / \mathrm{mL}$ Hoechst 33342 (SigmaAldrich) for 90 minutes at $37^{\circ} \mathrm{C}$. Cells were then washed with ice-cold HBSS and labeled with CD3-APC for flow cytometry analysis. To detect the side population (SP) cells, Hoechst 33342 samples were excited using a $355 \mathrm{~nm}$ UV laser on a LSRII/Fortessa flow cytometer (BD Biosciences; refs. 21, 22).

\section{Cytokine measurements}

Culture supernatants were collected after 24 hours of coculture to measure the release of IL2, IFN $\gamma$, and TNF $\alpha$, and after 72 hours for IL15, using specific ELISA (R\&D Systems, Inc). Plasma obtained from mice was analyzed for the presence of human cytokines using the Bio-Plex Pro Human Cytokine 8-plex Assay (Bio-Rad) or Human High Sensitivity Cytokine B Premixed Mag Luminex Performance Assay (R\&D Systems) following the manufacturer's instructions. Data were collected and analyzed using the Lumina-200 System and the Bio-Plex Manager 6.1 software (Bio-Rad).

\section{Activation of the suicide gene}

The B/B homodimerizer AP20187, a chemical inducer of dimerization drug (CID; Clontech Laboratories), was added at $50 \mathrm{nmol} / \mathrm{L}$ to T-cell cultures and induction of apoptosis evaluated 24 hours later using Annexin-V/7AAD staining (BD Biosciences) and FACS analysis (16).

\section{Immunophenotyping}

Cells were acquired using the BD FACS Fortessa (BD Biosciences) and analyzed using the FlowJo Software (Tree Star). mAbs specific for GD2, CD45, CD3, CD8, CD45RA, CD45RO, PD-1, CCR7, CD45, LAG-3, TIM-3, TIGIT, CTLA-4, CD25, CD69, active caspase-3, and granzyme $\mathrm{B}$ were obtained from BD Biosciences. To detect the GD2.CAR expression in transduced T cells, we used the $1 \mathrm{~A} 7$ anti-idiotype mAb specific for the $14 \mathrm{G} 2 \mathrm{a} . \mathrm{scFv}$ and PE or APC-conjugated rat anti-mouse secondary mAbs (BD Biosciences; ref. 5). In selected experiments, counting beads (Count Bright, Invitrogen) were added following the manufacturer's instructions. For the intracellular staining, cells were stained with Zombie dye and surface antibodies, including CD3 and GD2, washed, fixed, and permeabilized with Cytofix/ Cytoperm (BD Biosciences), and then stained with active caspase3 in $1 \times$ permeabilization buffer, following the manufacturer's instructions.

\section{Xenogeneic neuroblastoma models}

Mouse experiments were performed in accordance with the University of North Carolina (UNC, Chapel Hill, NC) animal husbandry guidelines according to protocols approved by the UNC institutional animal care and use committee. For these studies, 6- to 8-week-old female and male NSG mice (NOD. $\mathrm{Cg}-P r k d c^{\mathrm{scid}} I L 2 \mathrm{rg}^{\mathrm{tm} 1 \mathrm{Wj1}} / \mathrm{SzJ}$, UNC Animal Studies Core Facility) received intravenously via tail injection $2 \times 10^{6}$ CHLA255 cells labeled with FFLuc. Tumor engraftment was measured as bioluminescence signal intensity (BLI) and expressed as total flux (p/s) using the in vivo IVIS imaging (Caliper Life Science; ref. 23). Two weeks after tumor engraftment, mice received intravenously either $2 \times 10^{6}, 5 \times 10^{6}$, or $10 \times 10^{6}$ control-Ts (Ctrl) or CAR-Ts. No exogenous cytokines were administered to the mice. Mice were bled at specific intervals (10-15 days, as per UNC-IACUC guidelines) to measure cytokines in the plasma and frequency of CARTs. Mice were followed after CAR-Ts treatment and euthanized when tumor growth caused discomfort as per veterinarian's recommendation. At the time of euthanasia, blood, spleen, liver, and bone marrow were isolated and analyzed to detect CAR-Ts. To assess differences in response to continuous exposure to tumor cells, we performed tumor rechallenge experiments, where tumorbearing mice were further injected intravenously with $3 \times 10^{6}$ CHLA255 cells labeled with FFLuc at day 7 and 14 or at day 28 after CAR-T treatment. In selected experiments, tumor-bearing mice received intraperitoneally the $\mathrm{PD}$-1-blocking antibody (clone EH12.2H7, BioLegend; $200 \mu \mathrm{g} /$ mouse every 4 days) starting from the day before the CAR-Ts infusion. In some experiments, mice were engrafted with the FFLuc CHLA255 tumor cell line genetically engineered to express high levels of PD-L1 (CHLA255-PD-L1 ${ }^{+}$cells) in the attempt to further promote T-cell exhaustion in vivo. To ensure the function of the suicide gene, $10 \times$ $10^{6}$ FFLuc-tagged CAR-Ts were injected intravenously into NSG mice. T-cell expansion was then tracked by in vivo imaging measuring T-cell BLI. Mice were randomized to receive vehicle or CID (AP20187, $50 \mu \mathrm{g} /$ mouse), and then imaging was performed to evaluate the elimination of T cells. In selected experiments, mice were challenged with $3 \times 10^{6} \mathrm{CHLA} 255$ cells, 4 days after CID/vehicle treatment.

\section{Statistical analysis}

ANOVA with Bonferroni correction and the two-sided unpaired $t$ test were used for comparison of 3 or more groups, or 2 groups, respectively, as stated in the figure legends. Mixed model ANOVA was applied to compare tumor growth in different groups of mice. Survival curves were plotted using the Kaplan-Meier method, and the differences in the survival between groups were assessed by log-rank test. Data are presented as mean \pm SD or SEM as stated in the figure legends. Statistical significance was defined at $P<0.05$. Statistical analysis was performed with Prism 5 (GraphPad Software).

\section{Results}

T lymphocytes transduced with a modified GD2.CAR expressing the IL15 retain a central memory/stem cell-like phenotype and show lower expression of inhibitory receptors

We first reevaluated the structure of the GD2.CAR generated using the scFv derived from the $14 \mathrm{G} 2 \mathrm{a}$ mAb. Specifically, we compared the previously constructed GD2.CARs encoding the CD28 endodomain (24) with a modified GD2.CAR, characterized by a shortened $\mathrm{V}_{\mathrm{H}}$ sequence and containing the hinge and transmembrane domain of human $\mathrm{CD} 8 \alpha$ instead of the Ig-derived hinge and CD28 transmembrane domain, and found that the new optimized GD2.CAR had superior activity in in vitro experiments (Supplementary Fig. S1A-S1F), and reduced selfdimerization (Supplementary Fig. S1G). We then generated two cassettes, encoding either the optimized GD2.CAR alone or the GD2.CAR in combination with IL15 (GD2.CAR.15) using a 2A-sequence peptide (Fig. 1A). CAR expression (Fig. 1B) and expansion (Fig. 1C) of T cells transduced with either GD2.CAR or GD2.CAR.15 vectors were similar. Phenotypically both GD2. CAR-Ts and GD2.CAR.15-Ts contained similar amounts of CD4 ${ }^{+}$ and $\mathrm{CD}^{+}{ }^{\mathrm{T}}$ cells (Supplementary Fig. S2A). In contrast, GD2. CAR.15-Ts contained higher frequency of central memory $\mathrm{T}$ cells 
A

\begin{tabular}{|c|c|c|c|c|c|c|}
\hline 5' LTR & scFv. $14 \mathrm{~g} 2 \mathrm{a}$ & hTM-CD8 $\alpha$ & CD28 & $\mathrm{CD} 3 \zeta$ & $3^{\prime}$ & \\
\hline 5' LTR & scFv.14g2a & hTM-CD8 $\alpha$ & CD28 & $\mathrm{CD} 3 \zeta$ & $2 A$ & IL15 \\
\hline
\end{tabular}
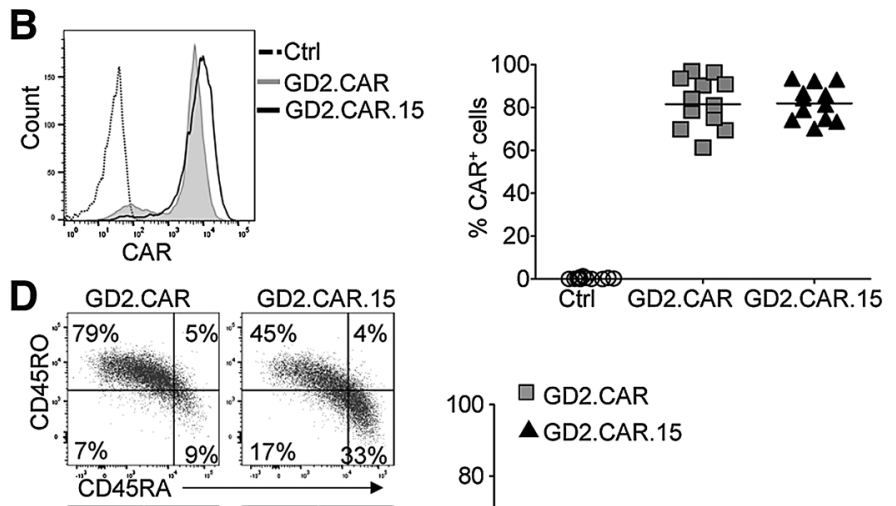

C
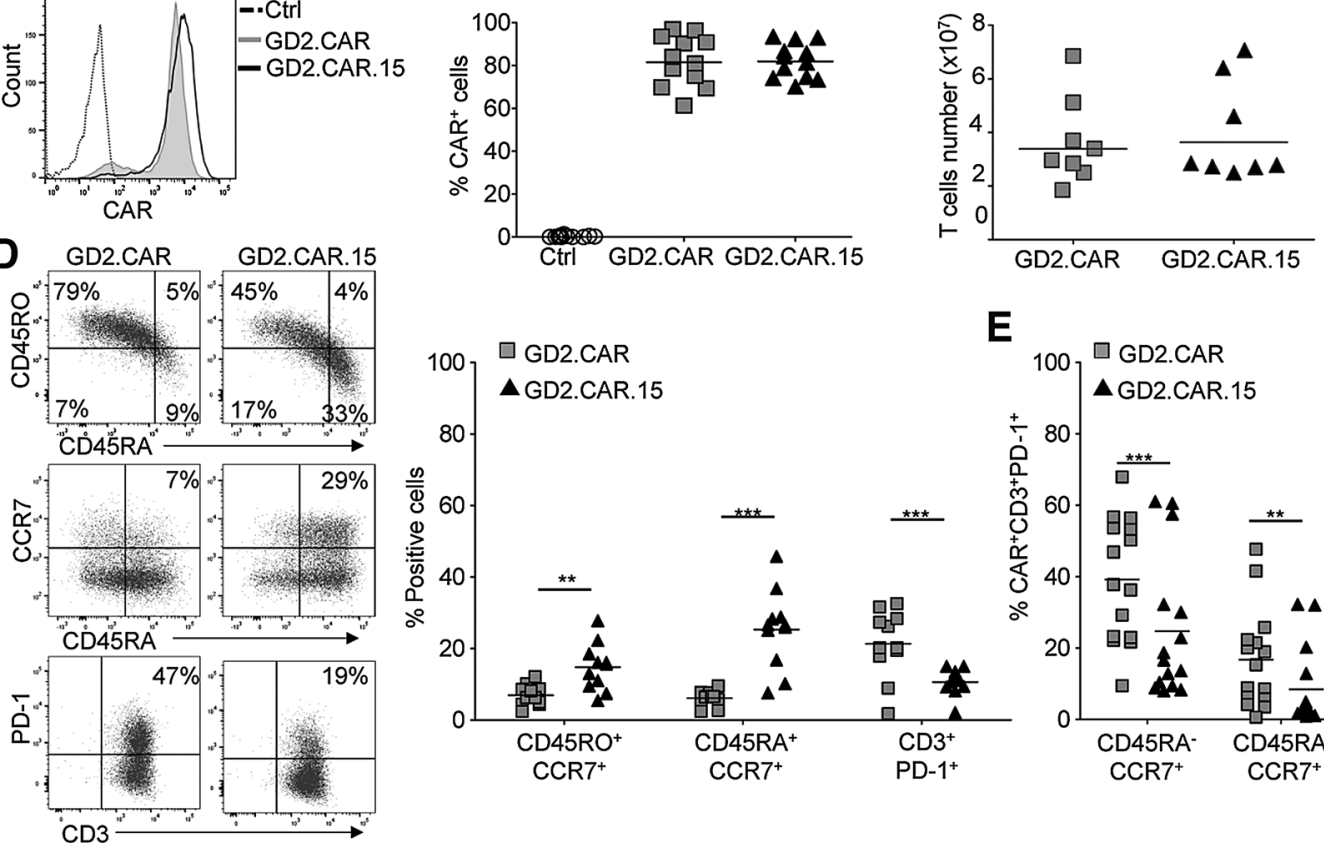

E

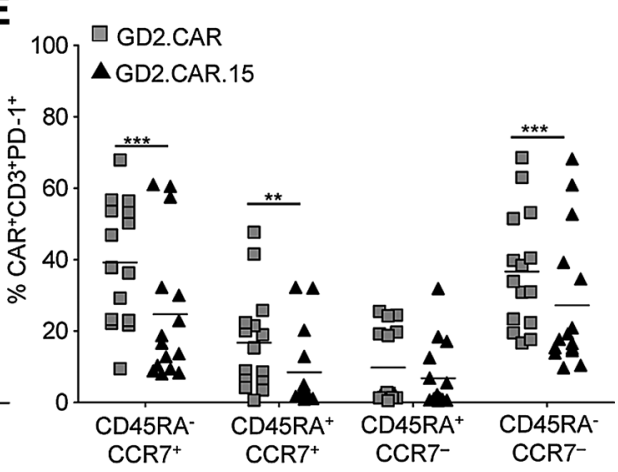

Figure 1.

Expression of GD2.CAR in activated T cells. A, Schematic representation of retroviral vectors encoding GD2.CAR with the CD28 endodomain with or without IL15. B, Flow cytometry histograms showing GD2.CAR expression in activated T cells for one representative experiment (left), and summary of all donors (right graph; $n=12$ ). Nontransduced T cells or mock vector T cells were used as a control. C, CAR-Ts expansion by day 11 after transduction ( $n=8)$. D, Representative flow plots showing CD45RA, CD45RO, CCR7, and PD-1 expression in CAR-Ts collected at day 11 after transduction (left), and summary of all donors (right; $n=11$ ). ${ }^{* *}, P<0.01 ;{ }^{* * *}, P<0.001$ by two-way ANOVA test. E, PD-1 expression in CD45RA and CCR7 subsets of CAR-Ts at day 11 after transduction $(n=15) .{ }^{* *}, P<0.01$; ***, $P<0.001$ by two-way ANOVA test.

$\left(\mathrm{CD} 45 \mathrm{RO}^{+} \mathrm{CCR} 7^{+} ; 15 \% \pm 7 \%\right.$ vs. $\left.7 \% \pm 3 \%, P<0.01\right)$ and stem cell-like T cells (CD45RA ${ }^{+} \mathrm{CCR}^{+} ; 25 \% \pm 11 \%$ vs. $6 \% \pm 2 \%, P<$ 0.001) than GD2.CAR-Ts (Fig. 1D). Furthermore, GD2.CAR.15-Ts showed lower constitutive expression of PD-1 as compared with GD2.CAR-Ts in both central memory $(25 \% \pm 20 \%$ vs. $39 \% \pm$ $17 \%, P<0.001)$ and stem cell-like T cells $(8 \% \pm 11 \%$ vs. $17 \% \pm$ $14 \%, P<0.01$; Fig. 1E; Supplementary Fig. S2B). Downregulation of PD-1 in GD2.CAR.IL15-Ts was confirmed at the transcription level (Supplementary Fig. S2C). In GD2.CAR.IL15-Ts, we also observed low expression of another inhibitory receptor, namely LAG-3 (Supplementary Fig. S2D), while the activation markers CD25 and CD69 were equally expressed in GD2.CAR-Ts and GD2.CAR.IL15-Ts (Supplementary Fig. S2E). Susceptibility to activation induced cell death (AICD) was also comparable between GD2.CAR-Ts and GD2.CAR.15-Ts (Supplementary Fig. S2F). Overall these data suggest that the low expression of PD-1 and LAG-3 in GD2.CAR.IL15-Ts does not reflect lower activation but rather a superior fitness. These data were corroborated in experiments showing that GD2.CAR.15-Ts proliferated and persisted longer in the absence of exogenous cytokines or antigen support in vitro (Supplementary Fig. S2G) and in vivo (Supplementary Fig. S3A and S3B), mimicking the advantage they will have in the circulation before engaging tumor cells.
GD2.CAR.15-Ts have improved antitumor activity and expansion upon antigen exposure in vitro

We cocultured GD2.CAR-Ts and GD2.CAR.15-Ts with four neuroblastoma cell lines (including MYCN-amplified neuroblastoma cells) at an E:T ratio of 1:1 (Supplementary Fig. S4A). Both GD2.CAR-Ts and GD2.CAR.15-Ts efficiently eliminated tumor cells (Fig. 2A and Supplementary Fig. S4B), including putative tumor stem cells identified as SP cells (Supplementary Fig. S4C). However, GD2.CAR.15-Ts proliferated significantly better in response to all neuroblastoma cell line tested (Fig. 2B). IL15 was detectable in the supernatant of cocultures with GD2.CAR.15-Ts, while no significant differences were observed for other cytokines, including IL2, TNF $\alpha$, and IFN $\gamma$ or granzymeB release as compared with GD2.CAR-Ts (Fig. 2C; Supplementary Fig. S4D and S4E). Moreover, as observed during the in vitro expansion with cytokines, without antigen stimulation, GD2.CAR.15-Ts showed lower expression of PD-1 (Fig. 2D) and LAG-3 (Supplementary Fig. S4F) upon coculture with neuroblastoma cells as compared with GD2.CAR-Ts. Target cells showed the same level of cleaved caspase-3, which is another marker of apoptosis, 24 hours post coculture (Supplementary Fig. S4G), indicating similar kinetic of cytotoxic activity of GD2.CAR-Ts and GD2.CAR.IL15-Ts. Remarkably, GD2.CAR.15-Ts showed sustained antitumor activity in stress coculture experiments (25) in which T cells were repetitively 

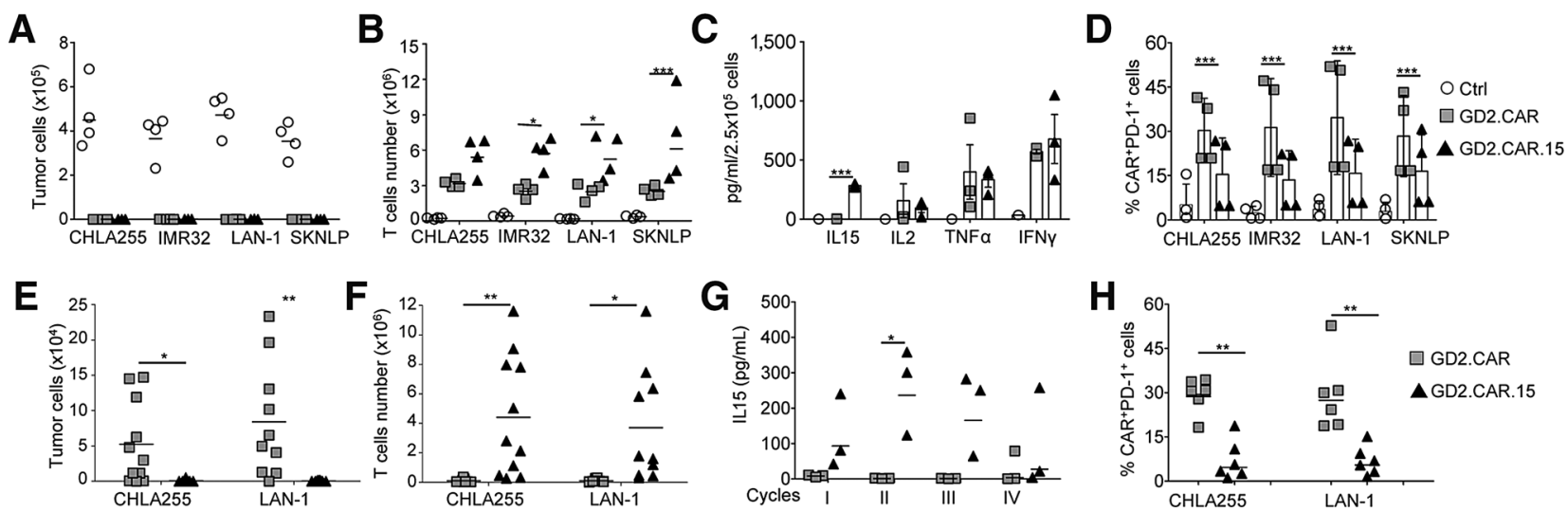

Figure 2.

GD2.CAR-Ts producing IL15 have enhanced cytotoxic activity upon repetitive exposure to tumor cells in vitro and produce Th1 cytokines. A and B, Quantification of residual tumor cells (A) and enumeration of T cells (B) in cocultures of control-Ts (Ctrl), GD2.CAR-Ts, or GD2.CAR.15-Ts with four GD2 ${ }^{+}$neuroblastoma tumor cell lines (CHLA255, IMR32, LAN-1, and SKNLP) at the E:T ratio of 1:1 for 7 days $(n=4) .{ }^{*}, P<0.05 ;{ }^{* * *}, P<0.001$ by two-way ANOVA test. C, Cytokine released in the coculture supernatant by Ctrl-Ts or CAR-Ts when cocultured with CHLA255 cells at the E:T ratio of 1:1. IL2, TNF $\alpha$, and IFN $\gamma$ were detected in supernatant collected 24 hours after the culture, whereas IL15 was detected after 72 hours $(n=4){ }^{* * *}, P<0.001$ by two-way ANOVA test. D, PD- 1 expression of Ctrl-Ts or CAR-Ts in culture with the indicated target cells at the E:T ratio of 1:1. Quantification of residual tumor cells (E) and enumeration of CAR-Ts (F) after four cycles of coculture of GD2.CAR-Ts or GD2.CAR.15-Ts with CHLA255 or LAN-1 cells $(n=10)$ at the E:T ratio of 1:1. ${ }^{*}, P<0.05$; ${ }^{* *}, P<0.01$ by two-way ANOVA test. G, IL15 released by GD2.CAR-Ts in response to CHLA255 cells after each cycle $(n=3) .{ }^{*}, P<0.05$ by two-way ANOVA test. H, PD- 1 expression by CAR-Ts after 4 cycles of coculture with CHLA255 or LAN-1 cells at the E:T ratio of 1:1 $(n=6) .{ }^{* *}, P<0.01$ by two-way ANOVA test.

exposed to antigen engagement using CHLA255 and LAN-1 cells (Supplementary Fig. S5A). After 4 cycles of coculture, GD2. CAR.15-Ts, but not GD2.CAR-Ts, significantly controlled tumor growth of both CHLA255 and LAN-1 cells $(P<0.05$ and $P<0.01$, respectively; Fig. 2E; Supplementary Fig. S5B) and proliferated in response to repetitive exposure to each respective tumor cell line $(P<0.01$ and $P<0.05$, respectively; Fig. 2 F). GD2.CAR.15-Ts continued to produce IL15 after each cycle of stimulation in

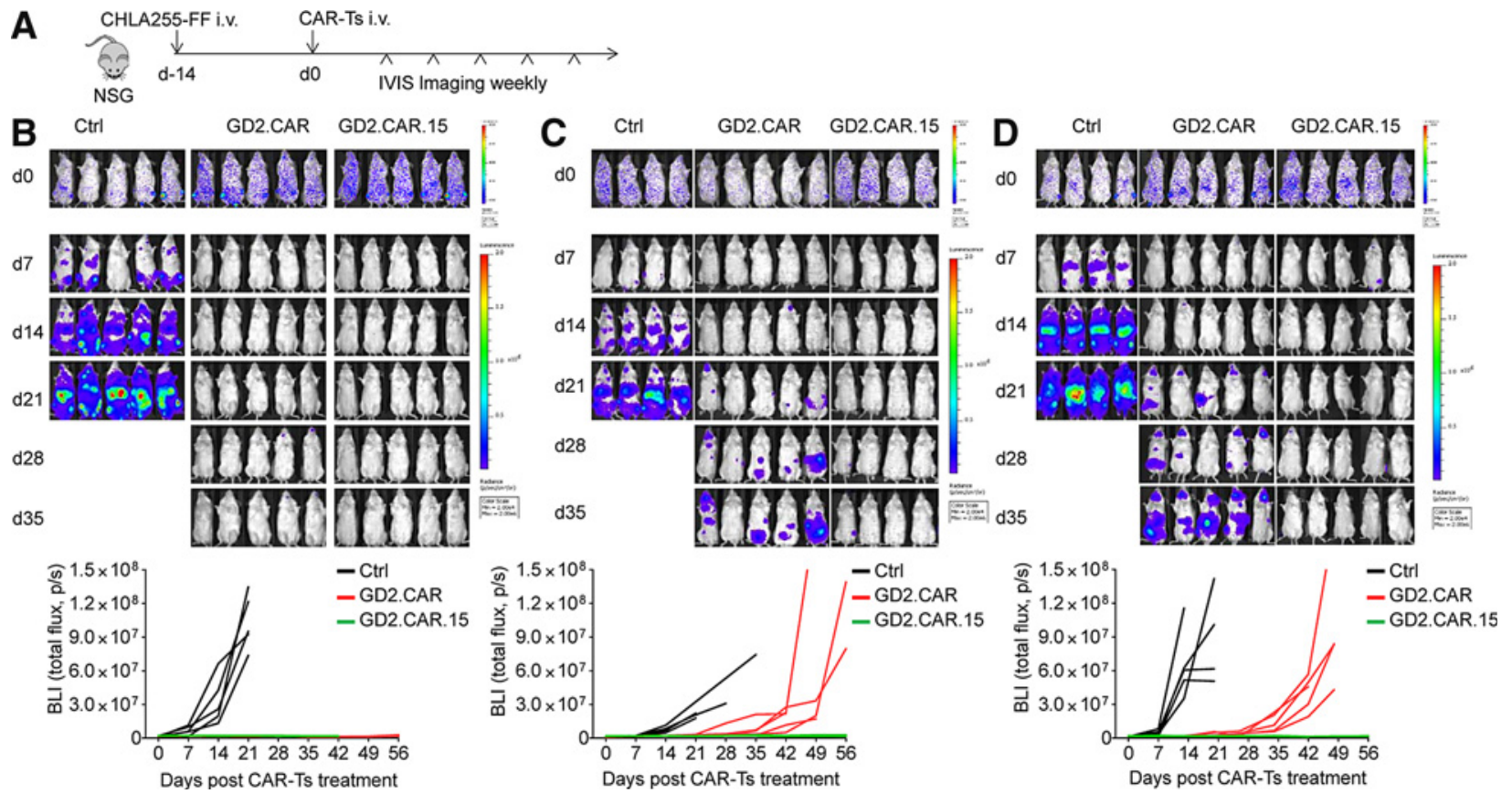

Figure 3.

Transgenic IL15 enhances antitumor effects of GD2.CAR-Ts in vivo. A, Schematic representation of the metastatic xenograft neuroblastoma model. NSG mice were inoculated intravenously via tail injection with $2 \times 10^{6} \mathrm{CHLA} 255$ cells labeled with Firefly-luciferase and two weeks later received T cells intravenously. B-D, Tumor growth in mice receiving $1 \times 10^{7} \mathrm{Ctrl}-\mathrm{Ts}$ or CAR-Ts (B), $5 \times 10^{6} \mathrm{Ctrl}$-Ts or CAR-Ts (C), or $2 \times 10^{6} \mathrm{Ctrl}$-Ts or CAR-Ts (D). Each panel shows images and bioluminescence (BLI) kinetics of tumor growth for each mouse ( $n=4-5 /$ group). 

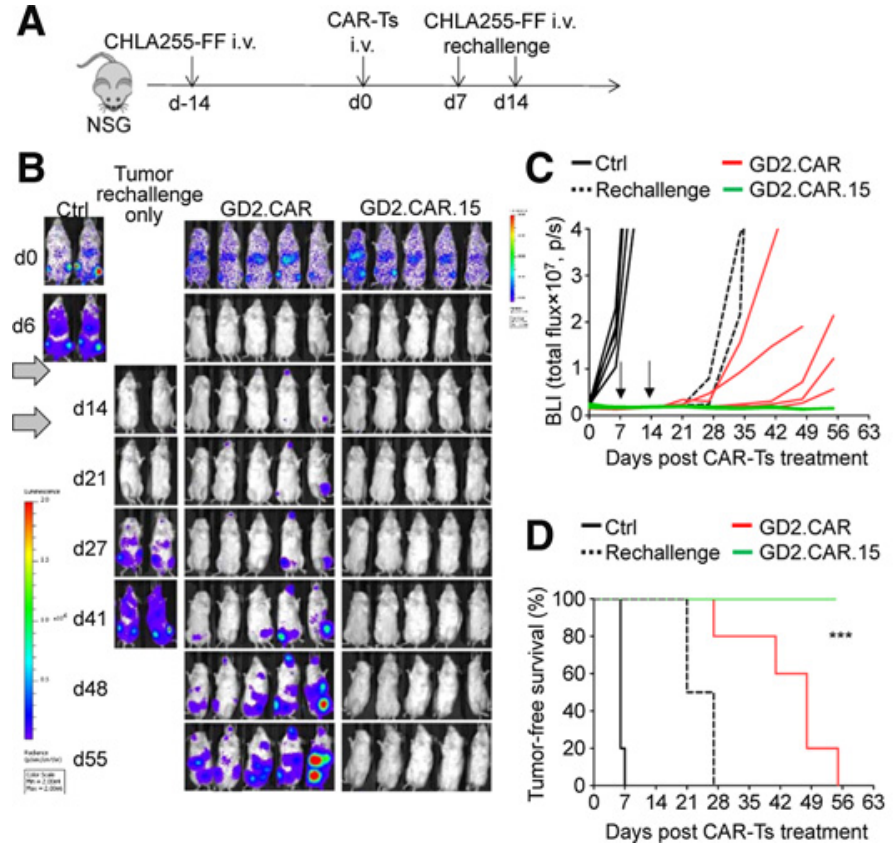
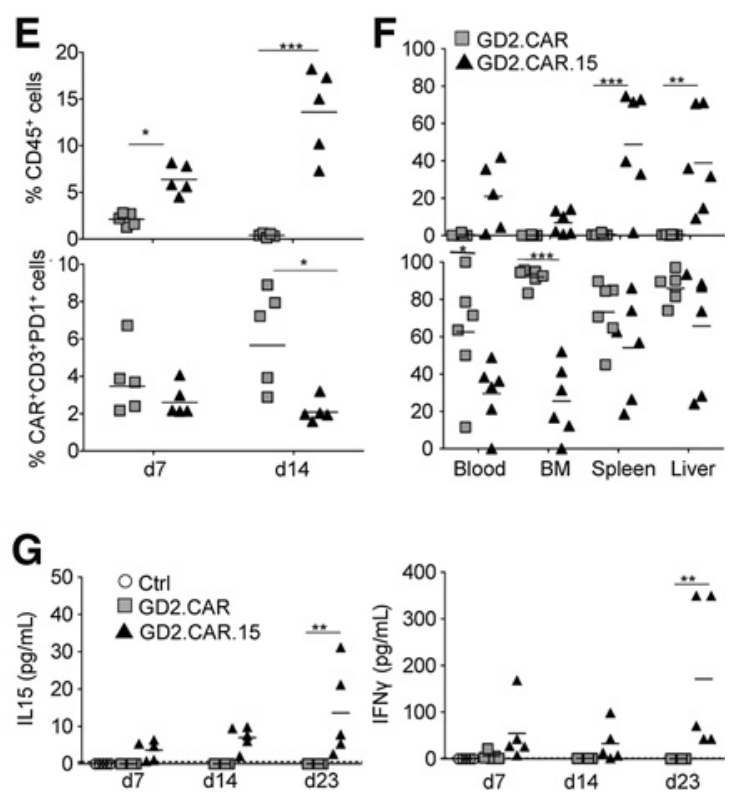

Figure 4.

Transgenic IL15 promotes antitumor activity and persistence of GD2.CAR-Ts in vivo in a rechallenge tumor model. A, Schematic representation of tumor rechallenge experiments in the metastatic xenograft neuroblastoma model. After tumor engraftment and T-cell treatment, mice received intravenous tumor CHLA255 cells labeled with Firefly-luciferase $\left(3 \times 10^{6}\right.$ cells/mouse) on days 7 and 14 . A group of mice infused with CHLA255 cells only was used as a second control for this model. B, BLI illustrating tumor growth in mice. Arrows indicate the tumor rechallenges. C, BLI kinetic of tumor growth. Each line represents an individual mouse for the $\mathrm{Ctrl}(n=5)$ or CAR-Ts treatment $(n=5)$ and rechallenge group $(n=2)$. Arrows, tumor rechallenges. D, Kaplan-Meier survival curve showing tumor free survival. Significance was determined by log-rank test (Mantel-Cox). ${ }^{* * *}, P=0.0005$. E, Quantification of human T cells (CD45 ${ }^{+}$cells; top) and $C A R^{+}$PD- $1^{+}$cells (bottom) in peripheral blood samples collected at days 7 and 14 after T-cell infusion $(n=5)$. ${ }^{*}, P<0.05$; ${ }^{* * *}, P<0.001$ by two-way ANOVA test. F, Quantification of human T cells (CD45 $5^{+}$cells; top) and CAR ${ }^{+} P D-1^{+}$cells (bottom) in peripheral blood, bone marrow, liver, and spleen at days 45 to 55 after T-cell treatment $(n=6)$. ${ }^{*}, P<0.05 ;{ }^{* *}, P<0.01 ;{ }^{* * *}, P<0.001$ by two-way ANOVA test. G, Detection of IL15 (left) and IFN $\gamma$ (right) in plasma samples collected at days 7,14 , and 23 after T-cell treatment $(n=5) .{ }^{* *}, P<0.01$ by two-way ANOVA test.

response to both CHLA255 (Fig. 2G) and LAN-1 (Supplementary Fig. S5C) cells. IFN $\gamma$ was released by CAR-Ts independently of the IL15 expression after the second round of stimulation with CHLA255 cells (Supplementary Fig. S5D), and upon repetitive antigen exposure, GD2.CAR.15-Ts continued to show lower PD-1 expression as compared with GD2.CAR-Ts (Fig. 2H). As observed after repetitive exposures to the tumor cells, CD25 and CD69 expression were similar between GD2.CAR-Ts and GD2.CAR. IL15-Ts (Supplementary Fig. S5E), and similar AICD was observed (Supplementary Fig. S5F). Overall, transgenic expression of IL15 harnessed significantly superior antitumor activity in an antigendependent fashion by promoting sustained T-cell proliferation after multiple exposures to tumor cells.

GD2.CAR.15-Ts are protective in an in vivo tumor rechallenge model. We compared the antitumor activity of GD2.CAR-Ts and GD2.CAR.15-Ts in a metastatic neuroblastoma model using NSG mice engrafted with the CHLA255 neuroblastoma tumor cell line (Fig. 3A). At the time of T-cell infusion mice were randomized based on the tumor BLI to ensure similar tumor burden between the experimental groups (Supplementary Fig. S6A). Tumor grew rapidly in mice treated with Ctrl cells, while GD2.CAR-Ts and GD2.CAR.15-Ts promoted equal control of tumor growth at day 50 when $1 \times 10^{7} \mathrm{~T}$ cells/mouse were infused (Fig. 3B). In contrast, only GD2.CAR.15-Ts succeeded in controlling tumor growth at day 50 when $5 \times 10^{6} \mathrm{~T}$ cells/mouse or $2 \times 10^{6} \mathrm{~T}$ cells/mouse were infused (Fig. 3C and D). The protective effect of GD2.CAR.15-Ts was even more prominent in tumor rechallenge experiments, when mice, in addition to the initial tumor burden, received weekly infusions of neuroblastoma tumor cells (Fig. 4A). GD2. CAR.15-Ts-treated mice were tumor free $>50$ days after T-cell infusion, while tumor growth was evident 2 weeks after the first tumor rechallenge in mice treated with GD2.CAR-Ts (Fig. 4B-D). GD2.CAR.15-Ts were detectable in the peripheral blood, and, consistently with the in vitro data, showed lower PD-1 expression (Fig. 4E). GD2.CAR.15-Ts were also detected in bone marrow, liver, and spleen at the time of euthanasia (Fig. 4F) and showed low PD-1 expression, although in these later stages they were

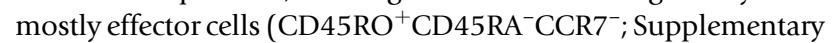
Fig. S6B-S6D). In addition to IFN $\gamma$, IL15 produced by GD2. CAR.15-Ts was detectable in the plasma up to day 23 postinfusion (Fig. 4G), while IL2 and TNF $\alpha$ were below the limit of detection (Supplementary Fig. S6E). To investigate whether the decreased PD-1 expression in GD2.CAR.15-Ts protects these cells in vivo from tumor induced exhaustion, tumor-bearing mice were treated with GD2.CAR-Ts or GD2.CAR.15-Ts in combination with the PD-1-blocking Ab (Supplementary Fig. S7A). PD-1 blockade did not provide any advantage in mice treated with GD2.CAR.15-Ts, because these cells show low PD-1 expression (Supplementary Fig. S7B). However, we also found that PD-1 blockade did not significantly improve persistence, cytokine release (Supplementary Fig. S7C and S7D), and antitumor activity (Supplementary Fig. S7B) in mice treated with CAR.GD2-Ts. When we 


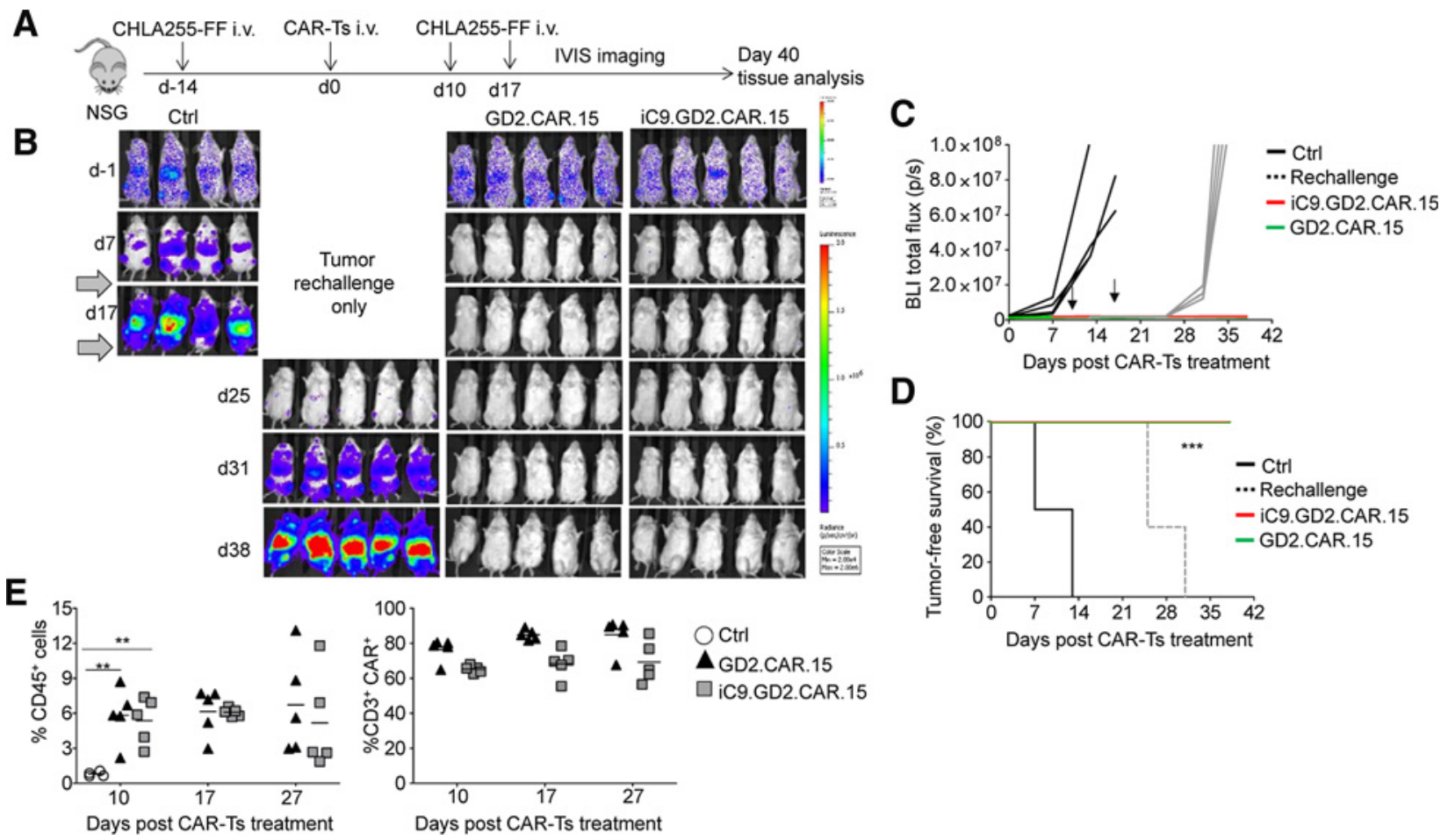

Figure 5.

iC9.GD2.CAR.15-Ts retain the same antitumor activity of GD2.CAR.IL15-Ts in vivo. A, Schematic representation of tumor rechallenge experiments in a metastatic xenograft neuroblastoma model. Upon tumor engraftment and T-cell treatment, mice received intravenous tumor CHLA255 cells labeled with Firefly-luciferase ( $3 \times 10^{6}$ cells/mouse) on days 7 and 14. A group of mice infused with CHLA255 cells alone was used as a second control for this model. B, BLI illustrating tumor growth in mice. Arrows, tumor rechallenges. C, BLI kinetic of tumor growth. Each line represents an individual mouse. Arrows, tumor rechallenge. D, Kaplan-Meier survival curve showing tumor free survival. Significance was determined by log-rank test (Mantel-Cox). ${ }^{* * *}, P<0.0001$. E, Quantification of human T cells (CD45 ${ }^{+}$cells; left) and $\mathrm{CD}^{+} \mathrm{CAR}^{+}$cells (right) in the peripheral blood of treated mice at days 10,17 , and 27 after T-cell infusions $(n=5) .{ }^{* *}, P<0.01$ by two-way ANOVA test.

used CHLA255 cells constitutively expressing PD-L1 (Supplementary Fig. S8A), GD2.CAR.15-Ts cells continued to control tumor growth with or without PD-1 blockade, while a modest advantage was observed with PD-1 blockade in mice treated with GD2.CARTs (Supplementary Fig. S8B-S8D). These data indicate that GD2. CAR.15-Ts persist better after tumor clearance and after tumor rechallenge, but these effects are not directly correlated with an improved resistance to PD-L1 inhibition.

An optimized GD2.CAR construct allows the accommodation of the iC9 safety switch, while preserving the functionality of IL15

Because of the potential for aberrant T-cell proliferation or toxicities in the presence of sustained exposure to IL15, we included the iC9 safety switch gene into our construct, to promptly remove GD2.CAR.15-Ts. Upon inclusion of the iC9 $c d n a$ into the GD2.CAR. 15 cassette, we unexpectedly observed a progressive loss of CAR expression when cells were maintained in culture, with a consequent reduced antitumor activity in vitro (Supplementary Fig. S9A). This phenomenon was a unique feature of the GD2.CAR, as a similar cassette constructed to express the CD19specific CAR did not show any significant reduction of the CAR expression (Supplementary Fig. S9B). Because the only difference in the two scFvs (CD19-specific and GD2-specific) was the size of the linker (20 versus 9 amino acids, respectively), we replaced the short-linker in the GD2-specific scFv with a long-linker (Supplementary Fig. S9C). Although the size of the linker did not significantly affect the expression and function of the GD2.CAR in the absence of the iC9 (Fig. 9D-F), the long-linker stabilized the GD2.CAR expression when the iC9 $c d n a$ was incorporated within the cassette (iC9.GD2.CAR.15; Supplementary Fig. S10A and S10B), allowing proper T-cell expansion in vitro (Supplementary Fig. S10C), and preservation of the same cell subset composition (Supplementary Fig. S10D). Expanded iC9.GD2.CAR.15-Ts showed functional activity of all three genes incorporated within the cassette. Specifically, the inclusion of the iC9 caused cell death when iC9.GD2.CAR.15-Ts were incubated with $50 \mathrm{nmol} / \mathrm{L}$ of the CID/AP20187 (Supplementary Fig. S10E). Moreover, iC9.GD2. CAR.15-Ts showed in vitro antitumor activity, proliferative capacity against CHLA255 or LAN-1 cells comparable with that of GD2.CAR.15-Ts (Supplementary Fig. S10F and Supplementary Fig. S11A and S11B), similar pattern of PD-1 expression (Supplementary Fig. S10G and Supplementary Fig. S11C), and similar release of cytokines (Supplementary Fig. S1OH and Supplementary Fig. S11D). Overall, an optimal design of the GD2.CAR allowed the functional accommodation of both the IL-15 and iC9 transgenes.

Using our rechallenge tumor model, we finally assessed in vivo the antitumor effects of iC9.GD2.CAR.15-Ts (Fig. 5A). Although tumor BLI rapidly increased in the control group, mice treated 
A

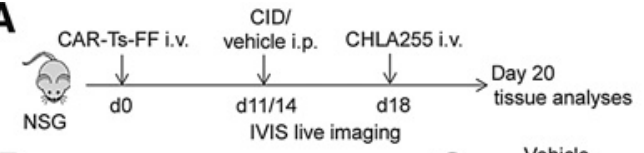

B
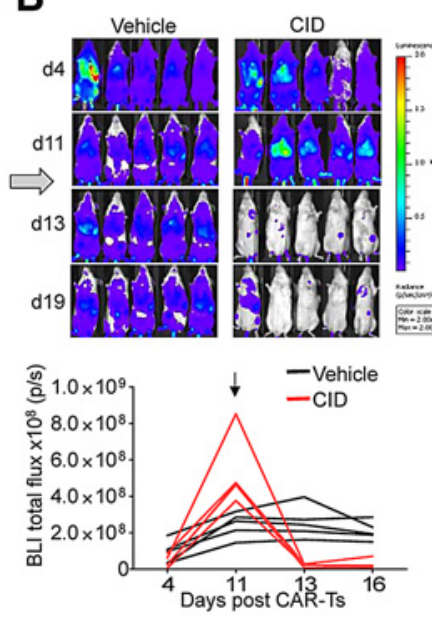

CID
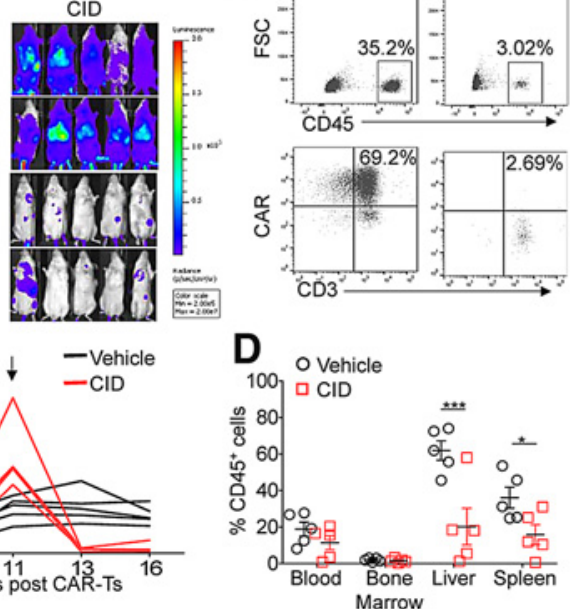
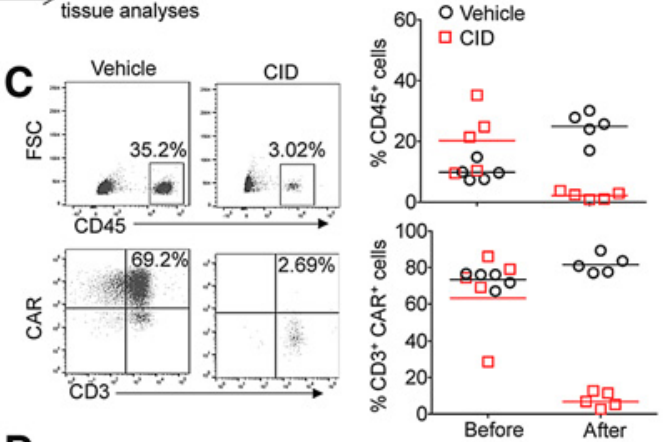

E
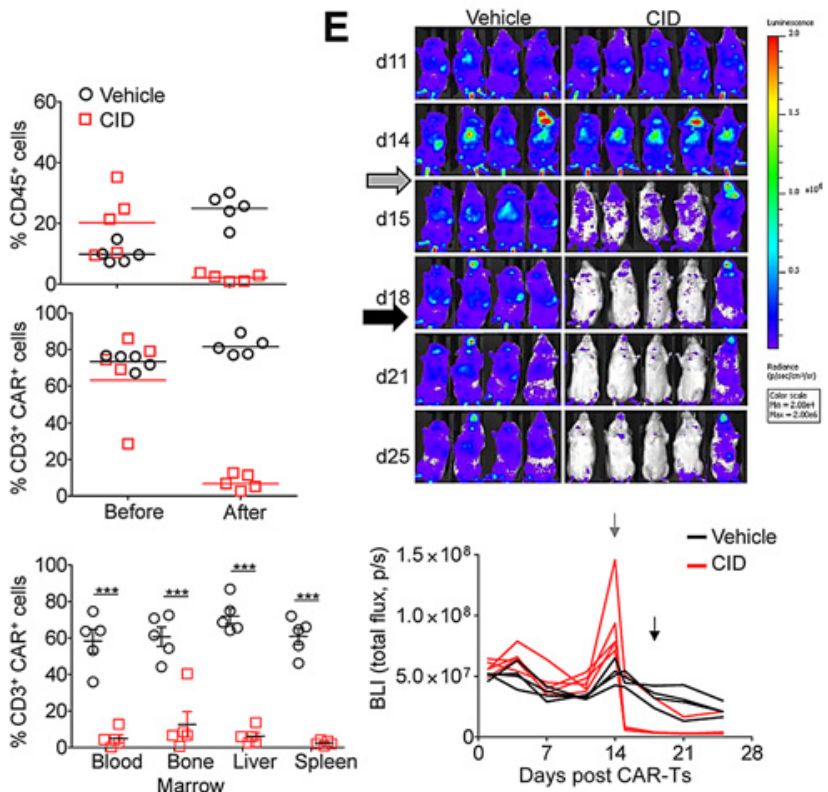

Figure 6.

iC9.GD2.CAR.15-Ts can be efficiently eliminated in vivo upon activation of the iC9 gene. A, Schematic representation of the experiments in NSG mice to test the iC9 activity in vivo. NSG mice were inoculated intravenously with T cells labeled with Firefly-luciferase. Mice received the vehicle or CID via intraperitoneal injection. In additional experiments, mice were inoculated intravenously with CHLA- 255 cells $\left(3 \times 10^{6}\right.$ cells/mouse $) 4$ days post CID with the aim to boost CAR-Ts that may have escaped CID-induced apoptosis. B, Images from representative mice before and after receiving either vehicle or CID as indicated by the arrow. The graph shows the BLI for of iC9.GD2.CAR.15-Ts in mice treated with either vehicle of CID as indicated by the arrow $(n=5)$. C, Representative flow plots and summary of human $\mathrm{CD}_{4} 5^{+}$and $\mathrm{CAR}^{+}$T cells in the peripheral blood before and after treatment with either vehicle or CID $(n=5)$. D, Summary of human $\mathrm{CD} 45^{+}$ and $\mathrm{CAR}^{+} \mathrm{T}$ cells in the peripheral blood and organs in the mice treated with either vehicle or $\mathrm{CID}(n=5) .{ }^{*}, P<0.05 ;{ }^{* * *}, P<0.001$ by two-way ANOVA test. E, Images from representative mice before and after receiving either vehicle or CID (gray arrow), and then rechallenged with tumor cells (black arrow). The graph shows the BLI of iC9.GD2.CAR.15-Ts in mice treated with either vehicle $(n=4)$ or CID $(n=5)$, and rechallenged with tumor cells.

with GD2.CAR.15-Ts and iC9.GD2.CAR.15-Ts remained tumor free by day 40 after rechallenge (Fig. 5B and C). This translated in a significantly better tumor free survival $(P<0.0001 ;$ Fig. 5D). GD2. CAR.15-Ts and iC9.GD2.CAR.15-Ts showed similar persistence (Fig. 5E) in mice after infusion, without significant differences in T-cell composition (Supplementary Fig. S12A). Together, our data show that the incorporation of the iC9 safety switch into an optimized GD2.CAR.15 construct remains beneficial in vivo.

To validate the functionality of the iC9 suicide gene, NSG mice infused intravenously with eGFP-FFLuc labeled iC9.GD2.CAR.15-Ts were treated with either AP20187 (CID $50 \mu \mathrm{g} /$ mouse) or diluent (vehicle; Fig. 6A). While progressively increasing in mice treated with vehicle, BLI decreased promptly and remained low after CID administration (Fig. 6B). Similarly, phenotypic analysis showed that circulating human $\mathrm{T}$ cells almost completely disappeared in CID-treated mice, while increased in vehicle-treated mice $(2 \% \pm 0.5 \%$ vs. $25 \% \pm$ $2 \%, P<0.001$; Fig. 6C). When we analyzed tissues collected at the end of the observation period, CAR-Ts were reduced in CID-treated mice as compared with vehicle-treated mice (Fig. 6D). Finally, in selected experiments when mice were rechallenged with tumor cells after CID or vehicle treatment to reactivate residual $\mathrm{T}$ cells, we observed no increase in BLI in any of the mice that previously received CID (Fig. 6E), which suggests that the residual CAR-Ts after CID treatment are not reactivated and thus cannot cause toxicity in vivo.

To further demonstrate the clinical applicability of the proposed approach, we have generated a clinical grade PG13 retro- viral packaging cell line (20) and produced clinical grade supernatant. As shown in Supplementary Fig. S13, iC9.GD2.CAR.15-Ts generated under GMP conditions for clinical use maintained the same characteristic of those generated in preclinical experiments ensuring the clinical application of the proposed strategy in patients with neuroblastoma. The clinical protocol was recently approved by FDA (IND 18500).

\section{Discussion}

GD2.CAR-Ts expressing costimulatory endodomains have been proven safe in patients with neuroblastoma, but their antitumor activity remains limited (4), suggesting that additional preclinical assessment is needed to improve their clinical activity. Here, we show that modifications of the GD2.CAR structure and the incorporation of IL15 proved to be key requirements to enhance antitumor activity without causing on target toxicity.

Our rationale for redesigning GD2.CAR-Ts stems from both laboratory and clinical observations. Laboratory observations have highlighted how structural characteristics of CAR molecules, such as the framework regions of the $\mathrm{scFv}$, and the length and origin of the hinge, and of the transmembrane endodomain, can impact the functionality of CAR-Ts (26-28). Specifically for the $\mathrm{scFv}$ of the GD2.CAR obtained from the $14 \mathrm{~g} 2 \mathrm{a} \mathrm{Ab}$, high tendency for self-aggregation secondary to interactions within the scFv framework regions has been described (27). This phenomenon was associated to an overstimulation of GD2.CAR-Ts, resulting in the premature exhaustion and reduced antitumor activity of these 
cells when the CAR contained the CD28 costimulatory signal (27). Furthermore, recent evidences indicated that a mutated version of the 14g2a.scFv (E101K), which enhances its binding affinity, although effective in controlling tumor growth, caused severe neurotoxicity in one study $(29,30)$, but not in another study (31). On the other hand, in patients with B-cell-derived malignancies the expansion of CD19-specific CAR-Ts in the peripheral blood correlates with objective antitumor activity (7). In contrast GD2specific CAR-Ts, although expanding in the peripheral blood when infused upon lymphodepletion, did not promote significant clinical benefits (4). On the basis of these observations for the $14 \mathrm{~g} 2 \mathrm{a}$-derived CAR, we sought to modify structural parts of our construct to address intrinsic limitations of the GD2.CAR cassette. In addition, we optioned for the inclusion of the IL15 cytokine as a survival factor for GD2.CAR-Ts rather than relying simply on PIK3 and TRAF mediated signaling delivered by the combination of CD28 or OX40/4-1BB endodomains upon antigen encounter $(24,32-35)$. We reasoned this would provide a cytokine signaling that is independent from the costimulation received, and from IL2 that is released by CAR-Ts upon antigen engagement $(32,35)$. We found that structural modifications of the GD2.CAR cassette reduced its spontaneous self-aggregation. Furthermore, upon incorporation of IL15, GD2.CAR-Ts retained specificity, but showed enhanced expansion and antitumor activities in vitro, after repeated tumor exposures, and in vivo in our innovative xenograft solid tumor model, which includes multiple tumor cell rechallenges to recapitulate the high tumor burden in patients with solid tumors. Importantly, these effects were obtained without evidences of "on target off tumor" toxicities.

Our study highlights that the inclusion of IL15 affords GD2.CAR-Ts with other important properties for translation into an effective therapy. First, the incorporation of the IL15 within the GD2.CAR produced an enrichment of cells with "memory" and "stem-cell" like phenotypes. This occurred during the expansion culture period, was independent from the specific antigen encounter by CAR-Ts, and was significantly superior to that already provided by the addition of exogenous IL15 and IL7 cytokines in the culture $(19,36)$. Although the mechanistic explanation of this phenomenon requires further studies, the immediate implication of this observation is that IL15 secreted by GD2.CAR-Ts promotes additional effects that are superior to those produced by soluble IL15. This may also be critical in patients, as the IL15 available in the plasma upon lymphodepletion, despite promoting T-cell expansion, may be inferior to the IL15 directly produced and released by GD2.CAR-Ts (4). Exogenous administration of IL15 is accompanied by systemic toxicities $(37,38)$, and thus we did not formally compare exogenous administration versus the endogenous production, as the former would not likely be clinically relevant. A membrane-bound form of IL15 expressed by CAR-Ts may be also beneficial (39). However, the incorporation of this gene would compromise the expression and function of the CAR, as the construct exceeds the cargo capacity of one single vector.

As second advantage, we observed is that GD2.CAR.15-Ts had significantly reduced expression of PD-1 and LAG-3 with and without antigen stimulation, and thus may be less susceptible to PD-L1-mediated inhibition, because neuroblastoma cells can express PD-L1, either constitutively or upon exposure to IFN $\gamma$ (Supplementary Fig. S8A). The observed low expression of PD-1 and LAG-3 in GD2.CAR.15-Ts is not related to a different activation status of these cells, but rather suggests their potential superior fitness upon multiple exposures to the antigen. The low expression of PD-1 in GD2.CAR.15-Ts correlated in vivo with lack of additive effects with PD-1 blockade in controlling the tumor growth. Of note, in our xenograft model of neuroblastoma, we also observed that PD-1 blockade did not improve the antitumor effects of GD2.CAR-Ts that express PD-1, and should be reinvigorated by PD-1 blockade. This outcome may be partially related to the murine model that does not allow sufficient time to establish T-cell exhaustion. However, our data resemble the lack of activity we have previously reported in a pilot clinical trial in patients with neuroblastoma (4). Additional studies are needed to clearly assess if PD-1/PD-L1 blockade can have a positive effect when combined with GD2.CAR-Ts in neuroblastoma. Nevertheless, our data indicate that GD2.CAR-Ts expressing IL15 remain effective even in a model in which we caused constitutive expression of PD-L1 in neuroblastoma cells.

Because IL15 is a T-cell growth cytokine, and GD2.CAR.15-Ts have more "stem-ness" properties, we incorporated a safety switch for regulation of potential unwanted and uncontrolled expansion $(16,17,40,41)$. Our data demonstrate that further CAR construction was required to functionally accommodate these three genes. However, upon optimization, the final vector cassette showed functionality in vivo, and we were able to scale up the production of the CAR-Ts for clinical use. This final step is important because complex CAR-T engineering strategies often fail to reach clinical applications due to difficulties in scaling up at GMP level the results obtained in preclinical experiments.

In conclusion, we demonstrate that GD2.CAR.15-Ts have functional properties that should provide enhanced antigenindependent persistence and improved antitumor activity. The clinical trial in patients with neuroblastoma based on the proposed strategy will be performed in our institution (IND $18500)$ and if proven safe and effective, it can have broader application for targeting solid tumors.

\section{Disclosure of Potential Conflicts of Interest}

G. Dotti reports receiving commercial research grants from Cell Medica and Bellicum Pharmaceutical and is a consultant/advisory board member for MolMed. No potential conflicts of interest were disclosed by the other authors.

\section{Authors' Contributions}

Conception and design: Y. Chen, G. Dotti, B. Savoldo

Development of methodology: Y. Chen, L. Metelitsa, G. Dotti, B. Savoldo Acquisition of data (provided animals, acquired and managed patients, provided facilities, etc.): Y. Chen, C. Sun, E. Landoni, G. Dotti

Analysis and interpretation of data (e.g., statistical analysis, biostatistics, computational analysis): Y. Chen, C. Sun, G. Dotti, B. Savoldo

Writing, review, and/or revision of the manuscript: Y. Chen, L. Metelitsa, G. Dotti, B. Savoldo

Administrative, technical, or material support (i.e., reporting or organizing data, constructing databases): Y. Chen, G. Dotti

Study supervision: Y. Chen, G. Dotti, B. Savoldo

\section{Acknowledgments}

This work was supported by UNC UCRF funds and in part by the Barnhill Family Foundation. Dr. Savoldo is supported by a NHLBI grant (R01HL114564) and a Hyundai Hope on Wheels Foundation grant.

The costs of publication of this article were defrayed in part by the payment of page charges. This article must therefore be hereby marked advertisement in accordance with 18 U.S.C. Section 1734 solely to indicate this fact.

Received June 12, 2018; revised November 30, 2018; accepted January 4, 2019; published first January 7, 2019. 


\section{References}

1. Maris JM. Recent advances in neuroblastoma. N Engl J Med 2010;362: 2202-11.

2. Maris JM, Hogarty MD, Bagatell R, Cohn SL. Neuroblastoma. Lancet 2007; 369:2106-20

3. Yu AL, Gilman AL, Ozkaynak MF, London WB, Kreissman SG, Chen HX et al. Anti-GD2 antibody with GM-CSF, interleukin-2, and isotretinoin for neuroblastoma. N Engl J Med 2010;363:1324-34.

4. Heczey A, Louis CU, Savoldo B, Dakhova O, Durett A, Grilley B, et al CAR T cells administered in combination with lymphodepletion and PD-1 inhibition to patients with neuroblastoma. Mol Ther 2017;25: 2214-24.

5. Pule MA, Savoldo B, Myers GD, Rossig C, Russell HV, Dotti G, et al Virus-specific $\mathrm{T}$ cells engineered to coexpress tumor-specific receptors: persistence and antitumor activity in individuals with neuroblastoma. Nat Med 2008;14:1264-70.

6. Louis CU, Savoldo B, Dotti G, Pule M, Yvon E, Myers GD, et al. Antitumor activity and long-term fate of chimeric antigen receptor-positive T cells in patients with neuroblastoma. Blood 2011;118:6050-6.

7. Maude SL, Frey N, Shaw PA, Aplenc R, Barrett DM, Bunin NJ, et al. Chimeric antigen receptor $\mathrm{T}$ cells for sustained remissions in leukemia. N Engl J Med 2014;371:1507-17.

8. Lee DW, Kochenderfer JN, Stetler-Stevenson M, Cui YK, Delbrook C, Feldman SA, et al. T cells expressing CD19 chimeric antigen receptors for acute lymphoblastic leukaemia in children and young adults: a phase 1 dose-escalation trial. Lancet 2015;385:517-28.

9. Brentjens RJ, Davila ML, Riviere I, Cui YK, Delbrook C, Feldman SA, et al. CD19-targeted T cells rapidly induce molecular remissions in adults with chemotherapy-refractory acute lymphoblastic leukemia. Sci Transl Med 2013;5:177ra38.

10. Kochenderfer JN, Dudley ME, Kassim SH, Somerville RP, Carpenter RO, Stetler-Stevenson $\mathrm{M}$, et al. Chemotherapy-refractory diffuse large B-cell lymphoma and indolent B-cell malignancies can be effectively treated with autologous $\mathrm{T}$ cells expressing an anti-CD19 chimeric antigen receptor. J Clin Oncol 2015;33:540-9.

11. Savoldo B, Ramos CA, Liu E, Mims MP, Keating MJ, Carrum G, et al. CD28 costimulation improves expansion and persistence of chimeric antigen receptor-modified $\mathrm{T}$ cells in lymphoma patients. J Clin Invest $2011 ; 121: 1822-6$

12. Liu D, Song L, Wei J, Courtney AN, Gao X, Marinova E, et al. IL-15 protects NKT cells from inhibition by tumor-associated macrophages and enhances antimetastatic activity. J Clin Invest 2012;122:2221-33.

13. Vera J, Savoldo B, Vigouroux S, Biagi E, Pule M, Rossig C, et al T lymphocytes redirected against the kappa light chain of human immunoglobulin efficiently kill mature B lymphocyte-derived malignant cells. Blood 2006;108:3890-7.

14. Rossig C, Bollard CM, Nuchtern JG, Merchant DA, Brenner MK. Targeting of G(D2)-positive tumor cells by human T lymphocytes engineered to express chimeric T-cell receptor genes. Int J Cancer 2001;94:228-36.

15. Quintarelli C, Vera JF, Savoldo B, Giordano Attianese GM, Pule M, Foster $\mathrm{AE}$, et al. Co-expression of cytokine and suicide genes to enhance the activity and safety of tumor-specific cytotoxic T lymphocytes. Blood 2007; 110:2793-802.

16. Straathof KC, Pule MA, Yotnda P, Dotti G, Vanin EF, Brenner MK, et al. An inducible caspase 9 safety switch for T-cell therapy. Blood 2005;105: 4247-54.

17. Diaconu I, Ballard $B$, Zhang $M$, Chen $Y$, West $J$, Dotti $G$, et al. Inducible caspase-9 selectively modulates the toxicities of CD19-specific chimeric antigen receptor-modified T cells. Mol Ther 2017;25:580-92.

18. Ramos CA, Ballard B, Zhang H, Dakhova O, Gee AP, Mei Z, et al. Clinical and immunological responses after $\mathrm{CD} 30$-specific chimeric antigen receptor-redirected lymphocytes. J Clin Invest 2017;127:3462-71.

19. Xu Y, Zhang M, Ramos CA, Liu E, Dakhova O, Liu H, et al. Closely related T-memory stem cells correlate with in vivo expansion of CAR. CD19-T cells and are preserved by IL-7 and IL-15. Blood 2014;123: 3750-9.

20. Ramos CA, Savoldo B, Torrano V, Ballard B, Zhang H, Dakhova O, et al. Clinical responses with $\mathrm{T}$ lymphocytes targeting malignancy-associated kappa light chains. J Clin Invest 2016;126:2588-96.
21. Hirschmann-Jax C, Foster AE, Wulf GG, Nuchtern JG, Jax TW, Gobel U, et al. A distinct "side population" of cells with high drug efflux capacity in human tumor cells. Proc Natl Acad Sci U S A 2004;101:14228-33.

22. Hong LK, Chen Y, Smith CC, Montgomery SA, Vincent BG, Dotti G, et al CD30-redirected chimeric antigen receptor T cells target $\mathrm{CD} 30(+)$ and CD30(-) embryonal carcinoma via antigen-dependent and Fas/FasL inter actions. Cancer Immunol Res 2018;6:1274-87.

23. Nishio N, Diaconu I, Liu H, Cerullo V, Caruana I, Hoyos V, et al. Armed oncolytic virus enhances immune functions of chimeric antigen receptormodified T cells in solid tumors. Cancer Res 2014;74:5195-205.

24. Pule MA, Straathof KC, Dotti G, Heslop HE, Rooney CM, Brenner MK A chimeric $\mathrm{T}$ cell antigen receptor that augments cytokine release and supports clonal expansion of primary human T cells. Mol Ther 2005;12: 933-41.

25. Hoyos V, Savoldo B, Quintarelli C, Mahendravada A, Zhang M, Vera J, et al. Engineering CD19-specific T lymphocytes with interleukin-15 and a suicide gene to enhance their anti-lymphoma/leukemia effects and safety. Leukemia 2010;24:1160-70.

26. Hudecek M, Lupo-Stanghellini MT, Kosasih PL, Sommermeyer D, Jensen $\mathrm{MC}$, Rader $\mathrm{C}$, et al. Receptor affinity and extracellular domain modifications affect tumor recognition by ROR1-specific chimeric antigen receptor T cells. Clin Cancer Res 2013;19:3153-64.

27. Long AH, Haso WM, Shern JF, Wanhainen KM, Murgai M, Ingaramo M, et al. 4-1BB costimulation ameliorates $\mathrm{T}$ cell exhaustion induced by tonic signaling of chimeric antigen receptors. Nat Med 2015;21:581-90.

28. Geldres C, Savoldo B, Dotti G. Chimeric antigen receptor-redirected T cells return to the bench. Semin Immunol 2016;28:3-9.

29. Richman SA, Nunez-Cruz S, Moghimi B, Li LZ, Gershenson ZT, Mourelatos $Z$, et al. High-affinity GD2-specific CART cells induce fatal encephalitis in preclinical neuroblastoma model. Cancer Immunol Res 2018;6:36-46.

30. Richman SA, Milone MC. Neurotoxicity associated with a high-affinity GD2 CAR-response. Cancer Immunol Res 2018;6:496-7.

31. Majzner RG, Weber EW, Lynn RC, Xu P, Mackall CL. Neurotoxicity associated with a high-affinity GD2 CAR-letter. Cancer Immunol Res 2018;6:494-5.

32. Dotti G, Gottschalk S, Savoldo B, Brenner MK. Design and development of therapies using chimeric antigen receptor-expressing T cells. Immunol Rev 2014;257:107-26.

33. Imai C, Mihara K, Andreansky M, Nicholson IC, Pui CH, Geiger TL, et al Chimeric receptors with 4-1BB signaling capacity provoke potent cytotoxicity against acute lymphoblastic leukemia. Leukemia 2004;18:676-84.

34. Finney HM, Akbar AN, Lawson AD. Activation of resting human primary T cells with chimeric receptors: costimulation from CD28, inducible costimulator, CD134, and CD137 in series with signals from the TCR zeta chain. J Immunol 2004;172:104-13.

35. Sadelain M, Brentjens R, Riviere I. The basic principles of chimeric antigen receptor design. Cancer Discov 2013;3:388-98.

36. Cieri N, Camisa B, Cocchiarella $F$, Forcato $M$, Oliveira $G$, Provasi $E$, et al. IL-7 and IL-15 instruct the generation of human memory stem T cells from naive precursors. Blood 2013;121:573-84.

37. Berger C, Berger M, Hackman RC, Gough M, Elliott C, Jensen MC, et al Safety and immunologic effects of IL-15 administration in nonhuman primates. Blood 2009;114:2417-26.

38. Conlon KC, Lugli E, Welles HC, Rosenberg SA, Fojo AT, Morris JC, et al. Redistribution, hyperproliferation, activation of natural killer cells and CD8 T cells, and cytokine production during first-in-human clinical trial of recombinant human interleukin-15 in patients with cancer. J Clin Oncol 2015;33:74-82

39. Hurton LV, Singh H, Najjar AM, Switzer KC, Mi T, Maiti S, et al. Tethered IL15 augments antitumor activity and promotes a stem-cell memory subset in tumor-specific T cells. Proc Natl Acad Sci U S A 2016;113:E7788-97.

40. Di Stasi A., Tey SK, Dotti G, Fujita Y, Kennedy-Nasser A, Martinez C, et al. Inducible apoptosis as a safety switch for adoptive cell therapy. N Engl J Med 2011;365:1673-83.

41. Zhou X, Dotti G, Krance RA, Martinez CA, Naik S, Kamble RT, et al. Inducible caspase-9 suicide gene controls adverse effects from alloreplete $\mathrm{T}$ cells after haploidentical stem cell transplantation. Blood 2015;125 4103-13. 